\title{
ANALYSIS OF VARIANCE IN THE ANALYTIC HIERARCHY PROCESS
}

\author{
Yujin Shen \\ The Stockton State College \\ Pomona, New Jersey, 08240 \\ I.S.A.
}

\begin{abstract}
.
In 197i. T. L. Santy developed the Analytic Hierarchy Process, which is widely applied in decision sciences. For each level of the hierarchy, a pairwise comparison matrix $A$ is made by each judge in a group and the weight vector is then derived from the matrix. Very" often, these weight vectors ase different from each other. This paper applies the multivariate analysis of variance to test if these weight vectors ape statistically different provided each judge can repeat his or her experiment several times.
\end{abstract}

\section{Introduction}

In the Analytic Hierarchy Process we very often have several judges to do pairwise comparisons for any $p$ given objects in a given level of the hierarchy and get several sets of weight vectors.

Due to sampling errors and differences among the judges, the sets of weight rectors thus derived might be different. Our concern is whether these differences in the observed sets of weight vectors are statistically significant. If they are not statistically significant, we can therefore assume that the observed differences anong the sets of weights are due to sampling etrors.

A way to attack this question is to use the method of multivariate analysis of variance of one way classification, which can be found in any standard multivariate textbook. We will follow in this paper the approach of Donald F. Morrison (1976) in his book, MJultivariate Statistical Methods.

\section{Theory and method}

Suppose there are $k$ judges and $p$ objects to be compared in a given level of the hierarchy. Each.judge represents one treatment in the aralysis of variance. The jth judge, $j=1,2 \ldots \ldots k$, is asked to do.$V$, pairwise comparisons independently. In many cases, this assumption of independence can be made true through experiment. Based on the results of the jth judge, we get $N$, eigenvectors, $\vec{w}_{1}, \ldots, \vec{w}_{N, 1}$, which are all the estimates of the true weight vector, where

$$
\vec{u}_{i}^{e}=\left(u_{1,1}, u_{i, 2}, \cdots, u_{1, p}\right)
$$

is the eigenvector of the pairwise comparison matrix made by the jth judge in his ith set of pairwise comparisons.

Define

for $\jmath=1 . \quad . k$

$$
\begin{aligned}
\vec{\mu}_{j}^{\prime}=E\left(\vec{u}_{i j}^{\prime}\right) & =\left(E\left(u_{1,1}\right), E\left(w_{1,2}\right), \cdot, E\left(u_{i, p}\right)\right) \\
& =\left(\mu, j, \mu_{j 3}, \ldots, \mu_{, p}\right)
\end{aligned}
$$

Our linear model can then be written as

$$
u_{z j l}=\mu_{\jmath l}-\epsilon_{0 j l}
$$


for $j=1, \ldots, k, i=1, \ldots, N, l=1, . \ldots p$, where $\epsilon_{1} l$ is the error term.

The null hypothesis we are interested in testing is

$$
H_{0}: \vec{\mu}_{1}=\vec{\mu}_{3}=\quad=\vec{\mu}_{k} \text {. }
$$

But notice that $\sum_{l=1}^{p} w_{1 j l}=1$, which implies that $\sum_{l=1}^{p} \mu_{j l}:=1$, for $\jmath=1, \ldots, k$. Therefore the null , hypothesis

$$
H_{0}: \vec{\mu}_{1}=\vec{\mu}_{2}=\cdots=\vec{\mu}_{k}
$$

is equivalent to the null hypothesis

$$
H_{0}:\left(\begin{array}{c}
\mu_{11} \\
\mu_{12} \\
\vdots \\
\mu_{1, j-1}
\end{array}\right)=\left(\begin{array}{c}
\mu_{31} \\
\mu_{22} \\
\vdots \\
\mu_{2,-1}
\end{array}\right)=\ldots=\left(\begin{array}{c}
\mu_{k 1} \\
\mu_{k 2} \\
\\
\mu_{k, p-1}
\end{array}\right)
$$

Thus we would drop the last component from all the eigenvectors $\vec{w}_{1}$, to test $H_{0}$.

Define

$$
\begin{aligned}
& \vec{y}_{i j}^{*}=\left(w_{1 j 1}, w_{1,2}, \cdots, w_{i j, p-1}\right), \\
& \vec{\varepsilon}_{z y}=\left(\epsilon_{i}, 1, \epsilon_{1,2}, \ldots \ldots, \epsilon_{1, p-1}\right) \text {, } \\
& Y=\left(\begin{array}{c}
\vec{y}_{11} \\
\vec{y}_{31} \\
\vdots \\
\vec{y}_{N_{1} 1} \\
\vdots \\
\vec{y}_{1 k}^{t} \\
\vec{y}_{3 k}^{t} \\
\vdots \\
\vec{y}_{N_{2 k}}^{t}
\end{array}\right) \text {. } \\
& E=\left(\begin{array}{c}
\vec{\epsilon}_{11}^{t} \\
\vec{\epsilon}_{21}^{t} \\
\vdots \\
\vec{\epsilon}_{N 1}^{t} \\
\vdots \\
\vec{\epsilon}_{1 k}^{t} \\
\vec{\epsilon}_{2 k}^{t} \\
\vdots \\
\vec{\epsilon}_{N k k}^{k}
\end{array}\right) \\
& \mathbf{x}=\left(\begin{array}{ccccc}
1 & 0 & \ldots & 0 & 0 \\
\vdots & \vdots & \ddots & \vdots & \vdots \\
1 & 0 & \ldots & 0 & 0 \\
0 & 1 & \ldots & 0 & 0 \\
\vdots & \vdots & \ddots & \vdots & \vdots \\
0 & 1 & \ldots & 0 & 0 \\
\vdots & \vdots & & \vdots & \vdots \\
0 & 0 & & 0 & 1 \\
& & & & \vdots \\
0 & 0 & & 0 & 1
\end{array}\right),
\end{aligned}
$$


there $X$ has been partitioned into $k N, \times k$ submatrices,

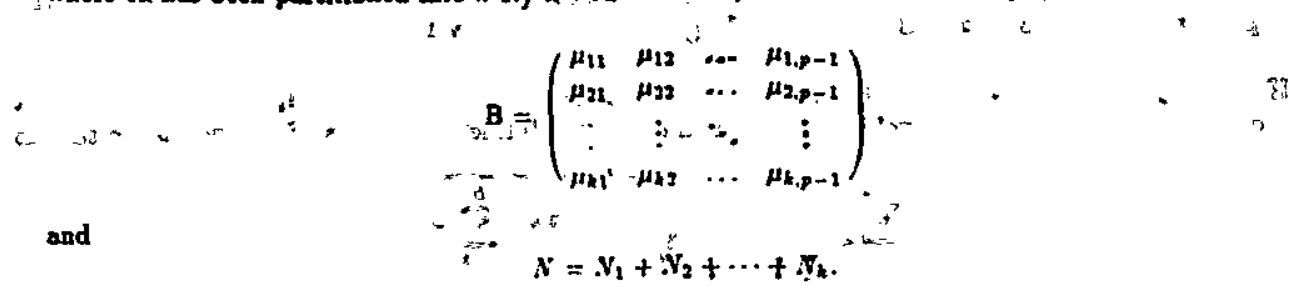

Then our linear model can be written as

$$
Y=x B+E .
$$

To apply the method of analysis of vatiance, we have to make the distribution assumption on the $\vec{y}_{i j}$ 's, namely, the $\vec{y}_{i j}$ 's have the independent normal distribution with mean vector $\left(\mu_{j 1}, \mu_{j 2}, \ldots, \mu_{2, p-1}\right)^{t}$ and common unknown covariance matrix $\Sigma$ for $j=1, \ldots, k, i=1, \ldots, N_{j}$.

To test the null hypothesis

$$
H_{0}:\left(\begin{array}{c}
\mu_{11} \\
\mu_{12} \\
\vdots \\
\mu_{1, p-1}
\end{array}\right)=\left(\begin{array}{c}
\mu_{21} \\
\mu_{22} \\
\vdots \\
\mu_{2, p-1}
\end{array}\right)=\cdots=\left(\begin{array}{c}
\mu_{k 1} \\
\mu_{k,} \\
\vdots \\
\mu_{k, p-1}
\end{array}\right)
$$

against the alternative hypothesis $H_{A}$ that at least two of the above mean veetors are different, we first compute the following two $(p-1) \times(p-1)$ matrices $H$ and $E$. The (rs)th element of $H$ is defined to be

$$
h_{r,}=\sum_{j=1}^{h} \frac{T_{y r} T_{j,}}{N_{j}}-\frac{1}{N} G_{r} G_{, .}
$$

The (rs)th element of $E$ is defined to be

$$
\text { " } \quad e_{r,}=\sum_{j=1}^{k} \sum_{i=1}^{N_{1}} w_{i j r} w_{i j,}-\sum_{j=1}^{k} \frac{T_{j,} T_{j,}}{\hat{N}_{j}}
$$

where

$$
T_{,} \cdot=\sum_{i=1}^{N_{j}} w_{i j,}
$$

and

$$
G_{,}=\sum_{j=1}^{k} T_{j}
$$

for $r=1,2, \ldots, p-1, s=1,2, \ldots, p-1$.

The $H$ matrix gives us the sum of squares due to the differenees among the judges and the $E$ matrix gives us the sum of squares due to the sampling errots.

We then caleulate the greatest eigenvalue $c_{3}$ of matrix $H E^{-1}$. Define $\theta_{s}=\frac{c_{1}}{i+c_{1}}$, with parameters $s=$ $\min (k-1, p-1), m=\frac{|k-p|-1}{2}$, and $n=\frac{N-k-1}{2}$ In Morrison 's book there is a distribution function table for the randorn variable $\theta$, with the above pnrnueters. The decision rule is as follows: If $\theta, \leq c_{a}^{*}$ accept 
$H_{0 ;}$ if $\theta_{0}>c_{0}^{*}$, rejecs $H_{0}$, where $c_{0}^{*}$ is the critical value found from the distribution table of $\theta_{a}$ inth the approtiate parameters $a, m$, and $n$ and with the given significance level $a$.

If $H_{0}$ is rejected at a level of significanci, we then would use the methods of multiple comparisons to get the $100(1-a)$ percent simultaneous confidence intervals on all lineas functions of the means contrast as follows:

$$
\begin{aligned}
& \sum_{n=1}^{p-1} \sum_{j=1}^{k} \alpha_{h} c_{j} \tilde{w}_{j h}-\sqrt{\frac{c_{a}^{*}}{1-c_{a}^{\alpha}} \bar{a}^{2} \vec{a}\left(\sum_{j=1}^{k} \frac{c_{j}^{2}}{N}\right)} \\
& \leq \sum_{k=1}^{p-1} \sum_{j=1}^{k} a_{k} c_{j} \mu_{j k n} \\
& \leq \sum_{h=1}^{n} \sum_{j=1}^{k} a_{h} c_{j} \tilde{u}_{j h}+\sqrt{\frac{c_{a}^{*}}{1-c_{a}^{*}} \vec{a}^{*} E a\left(\sum_{j=1}^{h} \frac{c_{j}^{2}}{N}\right)},
\end{aligned}
$$

where $\vec{w}_{j \mathrm{~A}}=\sum_{j=1}^{N_{j}} \frac{w_{j \mathrm{~A}}}{N_{2}}, \vec{a}^{*}=\left(a_{1}, a_{2}, \ldots, a_{p-1}\right)$ is any $p-1$ dimension vector, $\sum_{j=1}^{k} c_{j}=0$ and $c_{a}^{*}$ is the critical value of the distribution table of $\theta$, with parameters $s=\min (k-1, p-1), m=\frac{|k-p|-1}{2}$, and $n=\frac{N-k-2}{2+\cdots}$.

In particular, if $\vec{c}=\left(c_{1}, c_{2}, \ldots, c_{k}\right)$ is the vector with one in the jth position and negative one in the $(j+1)$ th position and sero elsewhere, we would have the $100(1-\alpha)$ percent simultaneous confidence interval for the lineas compound

$$
\sum_{h=1}^{p-1} a_{h}\left(\mu_{j h}-\mu_{j+1, h}\right)
$$

of the difference of the effects of judges $j$ and $j+1$.

3. Conclusion

This paper applied the multivariate analysis of variance to test if the weight vectors derived from several judges are statistically different. Multivariate one way analysis of variance seems useful in handling the variations due to the sampling errors and the differences among the judges. But it requires that each judge do repented pairwise comparisons over the objects independently, and hence the process takes more time. The assumption of multinormal distribution of the estimated weights seems to be questionable. When we have a balinced design, the test will be more powerful.

\section{REFERENCES}

1. Morrison, D. F. (1976), Multivatiote Statistical Methods, McGraw-Hill Book Company, New York.

2. Santy, T. L. (1980), The Analytic Fierarehy Process, MeGraw-Hill Book Company, New York.

3. Saty, T. I. (1977), "A Scaling Method for Priorities in Hierarchical Struetures." Jontanl of Mathematien! Parcholor 16, pp234-281. 